\title{
Haptic perception of object distance in a single-strand vibratory web
}

\author{
J. M. KINSELLA-SHAW and M. T. TURVEY \\ Center for the Ecological Study of Perception and Action \\ University of Connecticut, Storrs, Connecticut \\ and Haskins Laboratories, New Haven, Connecticut
}

\begin{abstract}
Can humans, like other animals, perceive distance by mechanical vibrations transmitted in a solid medium? In seven experiments, subjects perceived the distances from the hand of occluded metal disks attached to a taut nylon strand. Mechanical waves were initiated at the hand by the subject or at the disk by the experimenter. The results of Experiments 1 and 2 showed that perceived distance was linearly dependent on object distance with or without practice. The results of Experiments 3 and 4 revealed an inverse dependency of perceived distance on strand tension. In Experiment 5, a constant difference in perceived distance between vertical and horizontal strand manipulations was found. The results of Experiments 6 and 7 showed that distance was perceptible when the mechanical wave was not initiated by the subject. The informational basis for this haptic spatial ability was sought in the dynamics expressed by the one-dimensional wave equation, specifically, in the constants relating strand forces to strand motions.
\end{abstract}

For many species, spatial perception is based on patterns of mechanical vibration in the surfaces on which they stand and move. The desert scorpion (Paruroctonus mesaensis) locates burrowing prey within half a meter by the temporal structure and amplitude difference in compressional (horizontal) and Rayleigh (retrograde elliptical) wave fronts transmitted in the sand (Brownell, 1984). The ordinary spider locates prey in its web on the basis of the induced vibrations (Barrows, 1915; Burgess \& Witt, 1976; Parry, 1965; Szlep, 1964; Witt, 1975). The resonance characteristics of many webs are such that the frequency range of vibration (below $30 \mathrm{~Hz}$ and above $700 \mathrm{~Hz}$ ) produced by desirable prey, such as flies, is sustained and even amplified, whereas vibrations in the lower and higher ranges produced by insects that are problematic prey items for the spider, such as hard-shelled beetles or wasps, are attenuated (Walcott, 1963, 1969). In "social" spiders, there is a demonstrated ability to distinguish conspecifics from potential prey (Burgess, 1976, 1979; Burgess \& Witt, 1976; Witt, 1975). While most spiders are solitary, aggressive, and cannibalistic (Bristowe, 1958), social spiders live in communal web structures and not only tolerate each other but cooperate in predation. As with the webs of solitary spiders, the communal web of the

This research was supported in part by NSF Grant BNS 90-11013. It is based on a doctoral dissertation presented by the first author to the University of Connecticut. The conceptual and technical contributions of Robert Shaw, Gwen Gustaffson, and members of the University of Connecticut Physics Department are gratefully acknowledged. We would also like to thank Claudia Carello for her assistance with the figures. Correspondence should be addressed to the first author at the Center for the Ecological Study of Perception and Action, University of Connecticut, U-20, 406 Babbidge Rd., Storrs, Connecticut 06269-1020. social spider attenuates sine-wave vibrations above and below the frequency range of the spider's characteristic prey. Here, the selective attenuation has the additional consequence of allowing members of the colony to move freely about the web sheet without eliciting predatory responses by other colony members: the vibrations produced by spider locomotion are damped out (Burgess, 1975).

For an organism to perceive on the basis of mechanical waves in a solid medium (such as sand or fiber), it needs minimally an effector organ that (1) can maintain contact with the medium and (2) possesses the means to register the deformation of its tissues caused by the redistributing of forces in the effector as the wave propagates. Patently, arthropod legs equipped with lyriform organs (spiders) or slit sensilla (scorpions) meet these minimal criteria but so do mammalian limbs interpenetrated by mechanoreceptors. Can mammals-specifically, humansperceive distal objects on the basis of mechanical waves in a solid medium?

Haptic exteroperception in humans is well documented. People can perceive the length, orientation, and shape of a hand-held object by wielding it; the basis of these haptic spatial abilities is the inertia tensor, which is specific to the hand-wielded object system and is an invariant of the wielding dynamics (Burton, Turvey, \& Solomon, 1990; Pagano \& Turvey, 1992; Solomon \& Turvey, 1988; Solomon, Turvey, \& Burton, 1989a, 1989b; Turvey, Burton, Pagano, Solomon, \& Runeson, 1992). The haptic perceiving of properties of the local surface layout-such as horizontal and vertical distance from the hand (Carello, Fitzpatrick, \& Turvey, 1992; Chan \& Turvey, 1991) and the magnitudes of gaps between surfaces (Barac-Cikoja \& Turvey, 1991)-have also been demonstrated under conditions in which people tap and strike the layout with 
hand-held implements. These latter cases of haptic exteroperception have been shown to be tied to the invariants of the particular dynamics characterizing the probing. The broad capacity of humans to capitalize on the dynamics arising from contact with objects and nearby surfaces suggests that they may, like other animals, be able to exploit mechanical waves in a solid medium to perceive the whereabouts of distal objects.

The method for testing the proposed ability uses a "haptic web" consisting of a single strand of uniformly dense material. Both tension along the strand and the distance between the strand's anchor points are adjustable. These configuration parameters, along with the known constant elastic contribution of a single homogeneous strand of a given material (here, nylon), make the haptic web a vibratory medium amenable to systematic experimental variation. A person can stand adjacent to the strand and set it into vibration manually by simply shaking it. The position of the hand relative to the strand's anchors provides another configuration parameter. The sum of these configurations is an apparatus providing a low-dimensional, tunable vibratory array for which the mechanics and formal characterization, that of the "stretched string," are well known (e.g., Crawford, 1968; Morse, 1948).

\section{EXPERIMENTS 1 AND 2}

Can a transient vibratory array support in humans, as in other species, the haptic perception of object layout in the local environment? In Experiments 1 and 2, and the experiments that follow, the subject shook a taut strand and judged (without the benefits of seeing or hearing) the distance of an object attached to the strand. Experiments 1 and 2 are identical with the exception that subjects in Experiment 2 were given prior practice on the task.

\section{Method}

Subjects. The subjects were undergraduate students at the University of Connecticut who participated in partial fulfillment of introductory psychology course requirements. Eight participated in Experiment 1, and 6 participated in Experiment 2.

Apparatus and Materials. The apparatus is shown in Figure 1. Its central feature is a $264.5-\mathrm{cm}$ strand of densely braided nylon $(0.6 \mathrm{~cm}$ in diameter) stretched tautly between two stanchions bolted to a raised platform. The subject stood on the platform behind an adjustable partition (see Figure 1, lower left and right). The elevation of the strand was $100 \mathrm{~cm}$ above the platform and $114 \mathrm{~cm}$ above the floor. The line was anchored at one end to a hand-crank worm screw bracketed to a stanchion (Stanchion A) and run over an adjustable sleeve with a low-friction nylon pulley. At the opposite end of the platform, the line was run over another adjustable sleeve with a low-friction nylon pulley bolted to a second stanchion (Stanchion B) and then tied to a lever-arm counterweight. This counterweight assembly was bracketed to Stanchion B. In concert, the worm screw and counterweight allowed for the continuous adjustment and constant maintenance of tension on the strand.

Stanchions A and B consisted of two cold rolled steel pipes $167.5 \mathrm{~cm}$ high and $3.2 \mathrm{~cm}$ in diameter. These stanchions were set within steel pipe wells bolted onto two raised platforms measuring $122.8 \mathrm{~cm}$ wide $\times 61 \mathrm{~cm}$ long, at a height of $14.7 \mathrm{~cm}$ above the floor. The stanchion platforms were set at opposite ends on top of a single, larger base platform measuring $122.8 \mathrm{~cm}$ wide $\times 369.1 \mathrm{~cm}$
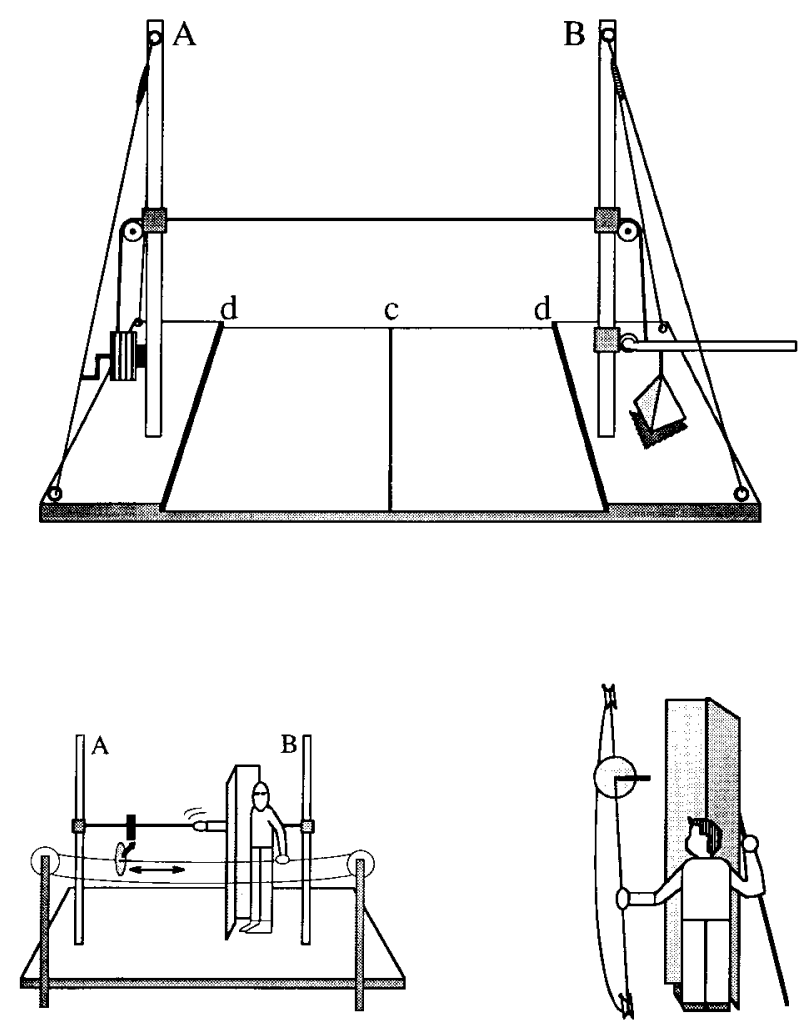

Figure 1. The minimal "haptic web" consisting of a strand of nylon under high tension (upper figure, see text for details). Lower left and right figures are views from vantage points from the side and from behind the subject, respectively. Strand is vibrated by occluded right hand, and report pointer is set by visible left hand.

long, elevated $12 \mathrm{~cm}$ above the floor. A 0.4-cm air space (a gap designated by the letter $c$ in Figure 1, upper panel) divided the superior surface of the base platform into two parts of equal length along a line beneath the midpoint of the taut strand. As a result, the superior surface of the base platform, in contrast to the strand, did not provide a continuous medium for the transmission of vibrations.

Each of the stanchion platforms rested upon a pair of $8 \times 8 \mathrm{~cm}$ steel squares and a pair of steel bars measuring $3 \mathrm{~cm}$ wide $\times 45 \mathrm{~cm}$ long. All of these steel pieces were cut from a single steel plate and had a thickness of $0.7 \mathrm{~cm}$. The stanchion platforms were positioned on top of the steel squares and bars at the ends of the base platform. The stanchion platforms, the steel support squares, and the underlying base platform were all aligned to achieve congruence of their edges at the corners of the base platform. Similarly, each steel bar was situated between a stanchion platform and the base platform, then aligned lengthwise with a lateral edge of each. Each steel support piece was bolted in place to the underside of one of the stanchion platforms. The end results of this arrangement were that (1) the stanchion platforms made contact only indirectly with the larger base platform and (2) air spaces of $0.7 \mathrm{~cm}$ (designated by the letter $d$ in Figure 1, upper panel) separated the platform surfaces, thereby minimizing the transmission of vibrations from the taut strand to the base platform upon which the subjects stood.

The stanchion platforms were fixed in position with lockable clamps. All platforms were constructed of 2 -cm-thick flooring-grade composition board bolted to building studs reinforced with angle iron. The hand-crank worm screw was fixed to Stanchion A with horseshoe pipe brackets $35.5 \mathrm{~cm}$ above the platform. The counter- 
weight lever-arm assembly on Stanchion B consisted of an aluminum $I$ beam upon which a 2-gal galvanized steel bucket containing $14.6 \mathrm{~kg}$ of lead was bolted. The entire counterweight assembly was attached to Stanchion $B$ with a hinged pipe bracket $50 \mathrm{~cm}$ above the platform. A cast metal adjustable pipe sleeve with a low-friction nylon pulley was bolted to each stanchion at a height of $95 \mathrm{~cm}$ above the platform. The pulleys on these sleeves demarcated the boundary points where impedance was maximal on the strand beyond which no wave can travel. The adjustable partition was constructed of two $1.27-\mathrm{cm}(1 / 2-\mathrm{in}$.) plywood sheets-one measuring $198 \mathrm{~cm}$ long $\times 61 \mathrm{~cm}$ wide, the second being of the same length but measuring $30.5 \mathrm{~cm}$ wide. These sheets were joined at $90^{\circ}$ along their lengths and reinforced with a building stud of the same length. This entire unit was then stood on end upon the platform.

The report apparatus stood parallel to the platform and at the same elevation above the floor as the taut strand. This apparatus consisted of a 616-cm nylon strand run in a loop between two lowfriction nylon pulleys bolted to two vertical poles set in bases directly on the floor $464.5 \mathrm{~cm}$ apart. The stanchions of the report apparatus were constructed of building studs fastened with bolts and nails. The upper part of the report loop passed through a disk (see Figure 1 , lower panels) of $10.5 \mathrm{~cm}$ in diameter cut from $.64-\mathrm{cm}(1 / 4-$ in.) plywood. A black arrow measuring $15 \mathrm{~cm}$ was attached to the disk on the report loop so as to point at the taut strand. Thus, the range over which the report arrow could be adjusted exceeded the length of the taut strand (from anchor points $A$ to $B$ ) by $1 \mathrm{~m}$ at either end of the platform.

The report loop was placed at a comfortably reachable distance, as indicated by each subject at the beginning of the experiment. A metal disk of $1.13 \mathrm{~kg}$ was attached to the taut strand with custommachined clamps that allowed for easy relocation along the strand and minimized eccentric motion by the object. Once situated upon the platform behind the partition, a subject could comfortably grasp both the taut strand and the report loop, but could see only the report loop and the attached disk-with-arrow. A retractable tape measure was run along the floor beneath the report loop and parallel to the taut strand's platform. The tape measure was not visible to the subject even when in use.

Finally, the $264.5-\mathrm{cm}$ strand of nylon was replaced after every 3rd subject to assure that any cumulative elastic fatigue effects did not alter the physical parameters of the medium.

Procedure. The subject was asked to determine haptically at what distance from his or her point of contact with the strand an object was attached to the line. In Experiments 1 and 2, the "point of contact" was the place at which the subject was instructed to grasp the strand, a point $61 \mathrm{~cm}$ from Stanchion B marked with a black dot. The subject was instructed to grasp the strand in a comfortable but secure grip with the thumb and index finger closing on the line, thus maximizing contact between the glabrous surfaces of the hand and the taut strand. When the hand was closed about the taut strand in this manner, the thumb-index-finger surface of the hand was oriented toward Stanchion $A$ and the attached object. The subject was then instructed to set the taut strand in motion ("get the line moving") in whatever manner he or she felt to be most effective. No further instructions or restrictions as to the amplitude, direction, or frequency of movement of the hand or taut strand were communicated to the subject.

Additionally, the subject was instructed to indicate where along the strand the object was attached by moving ("reeling in" or "reeling out') the report arrow to a position parallel to the object. The prescribed manner for adjusting the position of the report arrow was demonstrated for each subject. The subject was encouraged to take as much time as needed to get a feeling for how far down the line from the hand the object was attached. The initial position of the report arrow was at either extreme of the report loop's span, $1 \mathrm{~m}$ beyond either Stanchion A or Stanchion B. The initial position of the report arrow was randomized across trials for each sub- ject. Thus, the distance of the object from the hand was determined haptically, and the reported object distance was determined visually. Measurements of perceived object distance were obtained by dropping a plumb line from the report arrow onto the concealed tape measure and then reading off the distance from the subject's point of contact with the strand.

It is important to underscore that the report loop and the taut strand between Stanchions A and B ("the haptic web") supporting the attached mass were very different in their mechanical properties. The haptic web was assembled such that the distance from the hand of the test disk would remain fixed under even the most vigorous oscillations of the strand. Stanchions A and B, with their accompanying assemblages of adjustable-tension guy-wire supports, provided stable anchorages and fixed points of reflectance for the vibratory patternings engendered by the subject's manipulation of the taut strand. Tests conducted during the construction of the apparatus determined that this configuration would remain stable under loadings approximately five times greater than those that could be brought to bear by any subject in any of the experimental conditions. This was done to ensure both the safety of the subject and the unique correspondence between the test disk-to-hand distance and the generated vibratory patternings in each experimental condition.

Conversely, the report loop was assembled so that the report disk would change position with the minimal application of force parallel to the ground plane, by virtue of the low tension on the report loop and the use of low-friction pulleys in the construction of the report apparatus. Additionally, unlike Stanchions A and B of the haptic web, the uprights of the report apparatus were neither of sufficient mass to remain immobile nor bolted down (at a fixed distance apart) if a subject had deviated from the prescribed method ("reeling in or out") of adjusting the report arrow and instead oscillated the loop in a manner analogous to his or her exploratory manipulations of the test strand. In sum, while the construction of the report apparatus facilitated the movement of the report arrow, it would not support potentially informative oscillations of the report loop, even if a subject had chosen to covertly attempt such manipulations contrary to the explicit task instructions. (Throughout each trial, the subject was under the observation of the experimenter. It is also worth noting that, while the distance between the taut strand and the report loop was short enough to allow holding both simultaneously, many subjects chose to manipulate the taut strand first and then, after breaking contact with the test line, adjust the position of the report arrow.)

Lastly, the subject was told to release the taut strand as soon as the report arrow was positioned to her or his satisfaction and to look straight ahead at the partition until told to begin the next trial. This practice allowed the experimenter to move the object along the strand for the next trial location without detection by the subject. Pilot work revealed that subjects were readily able to detect any movement of the metal disk along the strand and to determine its direction, thus necessitating the requirement that the subject release the strand between trials.

The subjects had no opportunity to see the apparatus until after the experiment. Each subject was met at the laboratory entrance and guided, while oriented away from the apparatus, to a position behind the partition that occluded all portions of the laboratory except for the line of sight required for the visually guided adjustment of the report arrow. As an additional safeguard, the entire haptic web apparatus was covered with an opaque coverlet prior to the entry of each subject into the laboratory, thereby ensuring the concealment of both the haptic web's absolute length and its dimensions relative to the proportions of the entire experimental ensemble.

After receiving the instructions and being positioned comfortably on the base platform behind the partition, the subject donned a set of close-air industrial headphones. This was done to diminish 
any possibly distracting background noise from the cables and turnbuckles that serve to maintain the rigidity of the stanchions when the subject's exploratory manipulations set the strand in motion. Pilot work determined that a subject in position behind the partition on the apparatus platform could detect neither the presence of the experimenter nor the oscillations of the haptic strand when not in direct contact with the strand, while wearing the headphones. This fact, in concert with concern for the subjects' comfort and maintenance of attentional focus, motivated the decision not to use stereo headphones over which noise could be introduced. The headphones, however, did not prevent the subject from hearing the experimenter when addressed loudly. The subject was given no feedback during the course of the experiment. After the final trial, the subject was shown how well he or she performed on that trial. The subject was then given a debriefing as to the issues addressed by the experiment.

The preceding procedure was followed in Experiments 1 and 2. Prior to Experiment 2, however, the subjects were given eight practice trials in which they were allowed to see, on completion of each trial, where the object was attached along the strand. Object distances in the practice session differed from those of the experiment proper.

Design. The test object-the 1.13-kg metal disk-was attached to the haptic strand at one of five distances from the subject's point of contact with the strand. The five distances used were $30.5,61$, 91,122 , and $152 \mathrm{~cm}$. The object was presented seven times at each distance to each subject in completely randomized order. The experimental session lasted approximately $45 \mathrm{~min}$.

\section{Results and Discussion}

The mean perceived object distance at each actual object distance was calculated for each subject in both experiments. The individual subject means and the group means were then regressed on the actual object distances. As can be seen in Table 1, across individual subjects, the smallest amount of variance in perceived distance accounted for by actual distance was $78 \%$ and the largest amount of variance accounted for was $100 \%\left[r^{2}(4)=.78\right.$, significant at $p<.05]$. Simple linear regressions showing mean perceived object distance as a function of actual object distance for both experiments are presented

Table 1

Simple Regression of Perceived Distance on Actual Distance for the Subjects in Experiments 1 and 2

\begin{tabular}{cccc}
\hline Subject & Intercept & Slope & $r^{2}(5)$ \\
\hline \multicolumn{4}{c}{ Experiment 1} \\
1 & 37.59 & 0.77 & 0.98 \\
2 & 17.97 & 0.56 & 0.91 \\
3 & 29.18 & 0.78 & 0.98 \\
4 & 12.42 & 0.75 & 0.88 \\
5 & 9.30 & 0.80 & 0.97 \\
6 & 8.20 & 0.81 & 0.97 \\
7 & 44.21 & 0.65 & 0.96 \\
8 & 12.01 & 0.73 & 0.78 \\
& Experiment 2 & \\
1 & -4.01 & 0.91 & 1.00 \\
2 & 5.28 & 0.76 & 0.99 \\
3 & 12.14 & 0.66 & 0.98 \\
4 & -4.04 & 1.00 & 0.96 \\
5 & 16.54 & 0.89 & 0.97 \\
6 & 19.93 & 0.75 & 0.98 \\
\hline
\end{tabular}

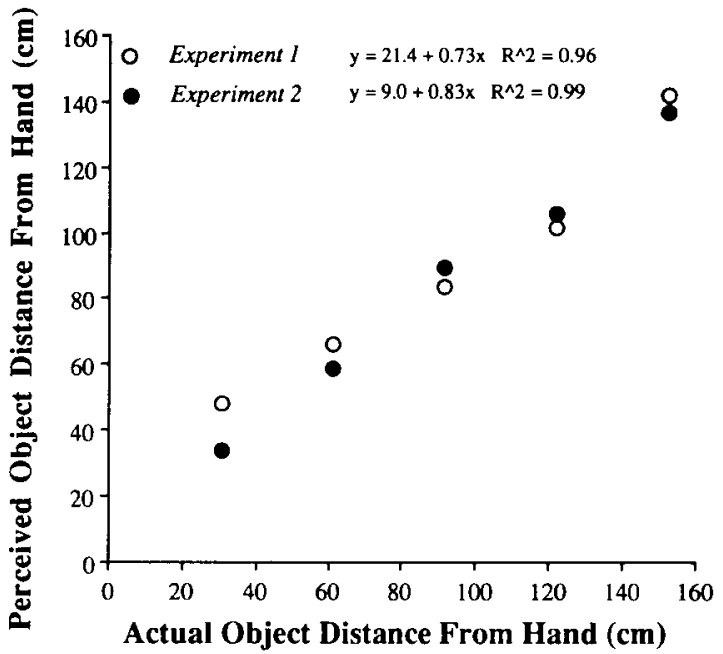

Figure 2. Regression lines for mean perceived distance against actual distance in Experiments 1 and 2.

in Figure 2. For actual distances of $30.5,61,91,122$, and $152 \mathrm{~cm}$, the mean perceived distances were 48.3, 66, 83.4, 101.6, and $141.6 \mathrm{~cm}$, respectively, in Experiment 1 , and $33.7,58.4,89.4,105.4$, and $136.2 \mathrm{~cm}$, respectively, in Experiment 2. Subjects apparently can perceive object distance along a solid medium when the medium is perturbed manually.

Combining the data provided by Experiments 1 and 2, an analysis of variance (ANOVA) revealed a significant main effect for the within-subject variable of object distance $[F(4,12)=215.11, p<.0001]$, but no effect for the between-subject variable of practice with feedback $(F<1$, with means of 34.7 and $32.7 \mathrm{~cm})$. The interaction of object distance and practice also proved to be nonsignificant $(p>.05)$. The ability to locate objects along a vibrating strand was unmodified by the availability of visual information about object distance during the practice session of Experiment 2. This result, however, does not rule out the possibility of improvement in this particular haptic spatial ability with more concentrated practice under visual confirmation. Improvement under such conditions is to be expected.

\section{EXPERIMENT 3}

Because the haptic system is stimulated only by forces that deform tissue, the wave quantity constraining distance perception must be some aspect of the propagating waves that reflects the vibrating forcings of the hand and yet, at the same time, is invariant over the frequency and amplitude variations in that forcing. The general strategy of much current research on haptic perception has been to identify and evaluate the parameters of the dynamical system characterizing a given haptic perception situation (e.g., Barac-Cikoja \& Turvey, 1991; Carello, Fitzpatrick, Domaniewicz, Chan, \& Turvey, 1992; Carello, Fitz- 
patrick, \& Turvey, 1992). These parameters are the constant quantities that couple the time-varying forces to the time-varying motions. For example, in wielding an object in order to perceive its length (e.g., Solomon \& Turvey, 1988), the inertia tensor is an invariant of the dynamics that couples the variable torques of exploration to the variable rotational motions that result.

When forces are imposed on a taut strand to initiate waves, the relation between the forces and the wave motions is given by the one-dimensional wave equation (Crawford, 1968; Morse, 1948; Symon, 1971)

$$
\partial^{2} u / \partial x^{2}=\left(1 / v^{2}\right)\left(\partial^{2} u / \partial t^{2}\right)
$$

where $u=u(x, t)$, a function locating each point $x$ on the string at every instant of time $t$, and $v$ equals $(T / \mu)^{1 / 2}$, with $T$ the tension and $\mu$ the linear density. The elastic force $\partial^{2} u / \partial x^{2}$ is coupled to the strand's motions $\partial^{2} u / \partial t^{2}$ by the constant $\mu / T$. If the density of the medium is always constant, then across different conditions of loading the strand-that is, placing an object of a given mass at different points along its length-the magnitude of $T$ will be the aspect in Equation 1 that uniquely characterizes the resultant wave propagation (see Appendix). $T$ is a parameter, therefore, that can be expected to be involved in the haptic spatial ability observed in Experiments 1 and 2 .

Experiment 3 pursued the strategy identified above by examining the effect of varying the mass of the object placed on the taut strand. Simply put, the question is, Will perceived object distance vary with object mass? Object weight can be expected to affect $T$. For a given distance, the larger the weight of the object at that distance, the larger the $T$ (see Appendix). Suppose that perceived distance varies inversely with $T$; given that $T$ increases with mass, perceived distance for a given hand position should be greater for lighter masses.

\section{Method}

Subjects. Nine undergraduate students at the University of Connecticut participated in partial fulfillment of introductory psychology course requirements. Two graduate student volunteers also participated, for a total of 11 subjects in Experiment 3.

Apparatus and Materials. The assembly and materials employed in this experiment were the same as those in Experiments 1 and 2 , with the exception that three disks were employed in this experiment weighing $.57,1.13$, and $2.27 \mathrm{~kg}$. A 1.13-kg disk had been used in the previous experiments.

Procedure. The procedure and the point of contact with the strand were identical to those of Experiments 1 and 2, with the modification that on any given trial, one of the three disks of different weights was used. Additionally, the disks were attached to the line at one of only three distances from the hand. The subjects were given no information as to the number and weights of the different disks used in the experiment.

Design. On a given trial, one of the three disks was attached at one of the following distances: $45.7,106.7$, and $167.6 \mathrm{~cm}$. Thus, there were nine object-distance configurations. Each subject was given each configuration seven times in an individually randomized order.

\section{Results and Discussion}

For the three masses, the mean perceived distances were numerically distinct and in concordance with the
Table 2

Partial Fs from Multiple Regression of Perceived Distance on Actual Distance and Object Mass in Experiment 3

\begin{tabular}{crll}
\hline Subject & Distance & Mass & $r^{2}(9)$ \\
\hline 1 & $48.19^{*}$ & 5.15 & 0.90 \\
2 & $155.56^{*}$ & 0.003 & 0.96 \\
3 & $95.97^{*}$ & 0.02 & 0.94 \\
4 & $51.19^{*}$ & $0.16 \dagger$ & 0.91 \\
5 & $227.63^{*}$ & $8.23 \dagger$ & 0.98 \\
6 & $1,010.32^{*}$ & 5.28 & 0.99 \\
7 & $47.42^{*}$ & 0.17 & 0.89 \\
8 & $53.08^{*}$ & 0.73 & 0.90 \\
9 & $42.02^{*}$ & 1.52 & 0.88 \\
10 & $59.05^{*}$ & 0.68 & 0.91 \\
11 & $53.50^{*}$ & 0.82 & 0.90 \\
\hline${ }^{*} p<.001$. & $+p<.05$. & &
\end{tabular}

expected inverse relation: $0.57 \mathrm{~kg}=111.5 \mathrm{~cm} ; 1.13 \mathrm{~kg}$ $=106.9 \mathrm{~cm} ; 2.27 \mathrm{~kg}=103.4 \mathrm{~cm}$. However, despite the systematic ordering of the means across the different object masses, an ANOVA revealed a marginally insignificant effect for the manipulation of object mass $[F(2,20)$ $=2.903, p=.07]$, whereas the main effect of actual object distance was highly significant $[F(2,20)=348.68$, $p<.0001]$. (For the object distances of 45.7, 106.7, and $167.6 \mathrm{~cm}$, the perceived distances were $69.3,103.9$, and $148.5 \mathrm{~cm}$, respectively.) The interaction between mass and actual distance also proved to be marginally nonsignificant $[F(4,40)=1.77, p=.07]$. As shown in Table 2, the multiple regression of perceived distance onto actual object distance and object mass for each individual subject shows that the partial $F$ for object mass was significant $(p<.05)$ for only 2 of the 11 subjects.

Although it had been expected that mass would affect perceived distance because of its necessary influence on $T$, the statistical analyses provided only marginal confirmation. As will become evident in the General Discussion section, a more direct assessment of $T$ reveals the expected contribution.

\section{EXPERIMENT 4}

In Experiment 4, a manipulation of tension that did not involve a change in the mass of the load applied to the strand was employed. Two different positions along the strand with maximally different tensions prior to loading were selected as the points of contact with the medium. To satisfy this requirement, the two points of contact selected were the center point of the strand and a point as near to Stanchion B (see Figure 1) as would allow for the free manipulation of the line by a subject. The tension $T$ is least at the center point of the unloaded strand and increases to its unloaded maximum at the anchor points on the stanchions. The two points selected were, therefore, distinguished in $T$ to the largest degree permitted by the task and apparatus.

When the object is attached at the same distances from each of the two selected points of contact (at the center of the taut strand and at the practicable end of the taut 
strand), it is necessarily attached at different positions along the strand. Imposing the weight of the object at different positions on the solid medium changes the tension along the portion of the strand that runs from the object through the point of contact to the anchor point on the stanchion. As noted above, a mechanical property of a taut strand is that $T$ is least at its midpoint and greatest at its anchor points. Thus, for grasping at the midpoint, $T \mathrm{~s}$ for an array of objects will be less, on the average, than for grasping at the ends. Again, suppose that distance perception relates inversely to $T$; perceived object distances will be greater, on the average, when the strand is grasped at its midpoint.

\section{Method}

Subjects. Ten undergraduate students at the University of Connecticut participated in partial fulfillment of introductory psychology course requirements. Each was assigned to his or her group by order of appearance at the laboratory.

Apparatus and Materials. The materials and the assembly were the same as those in Experiments 1 and 2.

Procedure. Each subject was randomly assigned to one of two groups; group membership determined at what distance from Stanchion B contact was made with the haptic strand. The partition between a subject and the haptic web was adjusted accordingly (see Figure 1, lower left). In all other aspects the protocol for Experiment 4 was identical to that for Experiments 1 and 2.

Design. Point of contact with the strand was a between-subject variable in Experiment 4 . In one group, the point of contact with the strand for a subject was $20 \mathrm{~cm}$ from Stanchion B. This was the point nearest to Stanchion B that would still permit the subject to manipulate the strand unimpeded by the support structures and moorings of the stanchion. In the other group, contact was made with the strand at the midpoint of the strand, $132.25 \mathrm{~cm}$ from Stanchion B. For a subject in either group, in each trial, the object was attached at one of four distances from his or her point of contact with the strand. Thus, while the distances from each respective point of contact were the same for each group, the points along the strand at which the object was attached were different. The four object distances from the two points where contact was made with the strand were $25.4,50.8,76.2$, and $101.6 \mathrm{~cm}$. A subject was presented with the 1.13-kg disk at each distance nine times in individually randomized order.

\section{Results and Discussion}

Figure 3 shows the simple linear regression for each subject of perceived distance against actual distance for contact at the end and middle of the strand. Inspection of Figure 3 suggests that the rate of change for perceived distance as a function of actual object distance was less when contact was made at the end of the strand than when contact was made at the strand's center. Additionally, inspection of Figure 3 suggests that perception was more accurate overall for the middle hand position. The slopes of the regressions reported in the lower right panel of Figure 3 proved to be significantly different beyond the $p=$ .0001 level $[F(1,4)=189.6]$ under a test for significant difference between the regression coefficients wherein the categorical variable of group membership is expressed in a coded vector (Pedhazur, 1982).
An ANOVA revealed no significant effect $(p>.05)$ for the between-subject variable of point of contact (mean perception for holding at end $=24.9 \mathrm{~cm}$ vs. mean perception for holding at center $=27.3 \mathrm{~cm}$ ); however, it did reveal a significant effect for the within-subject variable of actual object distance $[F(3,24)=137.04, p<.0001]$, together with a significant interaction between point of contact and real object distance $[F(3,24)=62.97$, $p<.0001]$. The significant interaction between the main variables obtained in Experiment 4 indicates changing hand position along the strand-such that the same object distances from the hand correspond to different locations along the strand under different tensions-profoundly influenced the perception of object distance from the hand. Because the same actual distances from the hand were perceived differently under different tensions, the outcome of this experiment supports the hypothesis that $T$ is the parameter that provides an index of the changes in wave structure upon which distance perception depends.

\section{EXPERIMENT 5}

At issue in Experiments 5-7 was the role of the subject's perturbation of the strand in the perceiving of object distances. Does the manner of the perturbation matter, and is self-initiated perturbation necessary for successful perception? The latter question was addressed by Experiments 6 and 7. Experiment 5 focused upon the issue of perturbation manner.

The effect on object distance perception of restricting a subject's exploratory manipulations of the strand to a single plane of motion was evaluated. Specifically, in Experiment 5 , the movements of a subject were constrained so as to lie exclusively in a plane either perpendicular or parallel to the ground plane and the surface of support provided by the apparatus platform. The experiment provides a basis for determining the relative importance of the contributions made by gravity and $T$ to the perceptually relevant properties of the wave structure engendered by shaking the strand. The one-dimensional wave equation has the form of Equation 1 when gravitational influences are deemed negligible. Such would likely be the case if the transverse wave motion was strictly parallel to the ground plane. If gravitational influences are deemed physically significant, then the one-dimensional wave equation takes the form

$$
\partial^{2} u / \partial x^{2}=\left(1 / v^{2}\right)\left(\partial^{2} u / \partial t^{2}\right)-g,
$$

where $g$ is the constant acceleration due to gravity (Crawford, 1968). If the transverse wave motion was restricted to a direction perpendicular to the ground plane, then a gravitational contribution might be expected.

Suppose that distance perception is linked to $v$, the quantity manipulated by variations in $T$ and $\mu$. If this is the case, then the results of Experiments 3 and 4 suggest that objects are perceived closer when $v$ is higher (or, synony- 

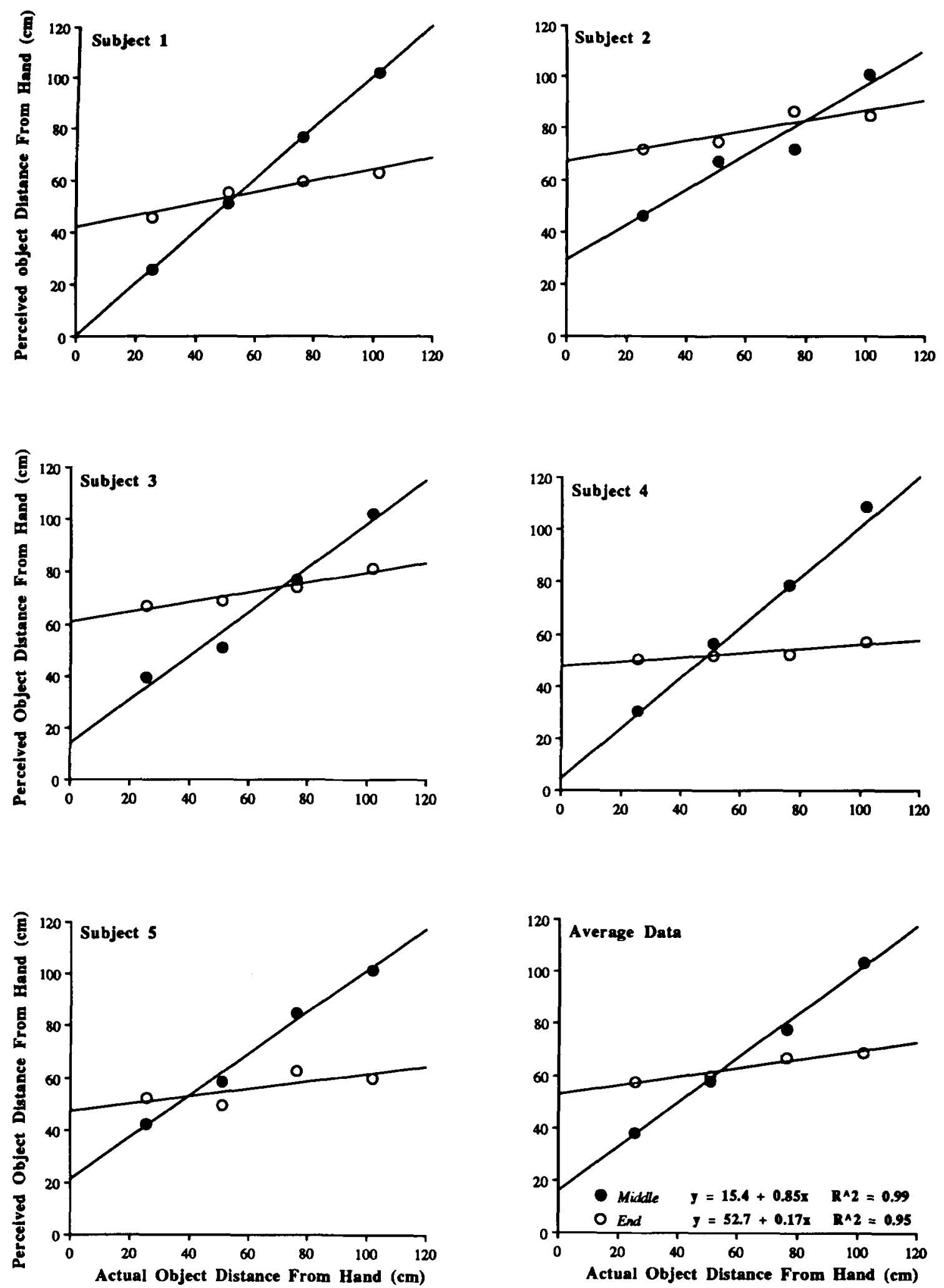

Figure 3. Regression of perceived distance against actual distance as a function of position of hand on the strand (at the end or in the middle) in Experiment 4. The regressions are shown for each of the individual subjects and for the averaged data. (In the subject panels, one regression line is for a middle-group subject and the other regression line is for an end-group subject. Subjects were paired in accordance with their order of appearance at the laboratory.) 
mously, when $T$ is larger). The one-dimensional wave equation can be rewritten so as to isolate $v$ as follows:

$$
v=\left[\partial^{2} u / \partial t^{2}\left(\partial x^{2} / \partial^{2} u+1 / g\right)\right]^{1 / 2} .
$$

From this form of the equation, it is clear that a gravitational contribution will result in slightly greater wave velocities. A comparison, therefore, of exploratory manipulations of the strand confined to either the vertical or the horizontal directions might yield distance perceptions that differ systematically but constantly over all actual object distances (reflecting the constant contribution, or lack thereof, of $g$ ). Explicitly, as $v$ is marginally greater for waves propagated by vertical motions of the strand, the perceived distances reported should be less than those reported for the same actual object distances when the direction of strand displacement is confined to the horizontal plane, if gravity has an effect on the perceptually salient properties of the mechanical wave.

Alternatively, when subjects are restricted to moving the strand solely in a horizontal direction relative to the ground plane, there is an additional component of tension added to the tensile state of the strand that is not encountered when explorations are confined to the vertical plane. This is a direct consequence of the requirement that the strand be displaced strictly parallel to the ground plane. If a force was applied to the attached object in a direction perpendicular to the strand and parallel to the ground plane, then the motions of the object would describe an arc-the strand-object system behaving as a simple gravity pendulum, like a child's swing.

In Experiment 5, however, the subjects were required to displace the strand linearly so that all motions remained in the horizontal plane, effectively moving the strandobject system along a trajectory that constitutes a chord across the arc that would be followed if the object were allowed to swing freely. To displace the strand horizontally, as required by the experimental task, a subject had to provide adequate force to overcome the radial component of force that contributes to the pendular trajectory followed by the freely swinging object (Halliday \& Resnick, 1974; Seto, 1964). In comparison with the tension encountered when movements are confined to the vertical direction, horizontal shaking will increase tension along the strand segment over which the mechanically propagated wave is transmitted to the hand. This would be a consequence of the fact that there is no radial component of force when movements are confined to the vertical direction, as the unimpeded motion of the strand-object system would follow a linear trajectory perpendicular to the ground plane. Simply stated, the tensions will be greater (for the same distances and hand position, with same mass object) when movements are confined to the horizontal plane than when movements are restricted to the vertical direction.

As a result of the addition of a radial component of force, the values for $T$ will be greater under horizontal explorations than under vertical explorations. From Equation 1 above, larger $T$ means higher $v$; thus, the velocity of waves generated by horizontal motions will be greater than the velocity of waves generated by vertical motions. Therefore, if perceived distance is linked inversely to $v$, then larger $T$ means shorter perceived distance. This leads to the prediction that manipulations of the direction of hand movements should be accompanied by variations in distance perception. Specifically, it follows that the distance from the hand to the object should be perceived as shorter for horizontal explorations than for vertical explorations, for the same actual object distances.

In sum, Experiment 5 provided an opportunity to evaluate competing hypotheses: If gravity contributes more to the perceptually significant wave component than to $T$, then perceived distances should be smaller under vertical movements than under horizontal movements; if $T$ contributes more to the perceptually significant wave component than to gravity, then perceived distances should be smaller under horizontal movements than under vertical movements.

\section{Method}

Subjects. Six undergraduate students at the University of Connecticut participated in partial fulfillment of introductory psychology course requirements.

Apparatus. The apparatus was identical to the assembly employed in Experiments 1 and 2, with the addition of a slotted template attached to the adjustable partition (see Figure 1, lower right). The template was oriented so as to intersect the single-strand haptic web in a plane both perpendicular and vertical to the horizontal span of the strand at the center of the template. This point coincides with the region where the rectangular slots in the template intersect. That is, taking the strand to run along the $x$-axis, the template lay in the $z y$-plane. In this coordinate system, the slots in the template restricted the direction of deflection of the strand to either the $z$ or the $y$ direction relative to the $x$-axis as defined by the horizontal span between Stanchions A and B.

Procedure and Materials. The procedure of Experiment 5 was the same as that of Experiments 1 and 2, with the addition that, prior to each trial, a placard bearing either a $V$ for vertical or an $\mathrm{H}$ for horizontal was displayed on the partition, instructing a subject as to which plane of motion he or she should restrict manipulations of the haptic strand. The movement template consisted of a square $(30.5 \times 30.5 \mathrm{~cm})$ piece of matte board into which two intersecting rectangular slots measuring $25 \times 2.54 \mathrm{~cm}$ were cut so that the point of intersection was located at the center of the template. The template was attached with finishing nails to a $100-\mathrm{cm}$ section of $1 / 4-$ in. $(.64-\mathrm{cm})$ dowel that was joined to the partition in a similar fashion. The placards indicating the permitted planes of motion consisted of $20.3 \times 27.9 \mathrm{~cm}$ white tagboard rectangles. All other materials were the same as those in Experiments 1 and 2.

Design. For either of the two planes of motion, the $1.13-\mathrm{kg}$ object was attached at one of four distances from the hand: $45.7,76.2$, 106.7 , or $137.2 \mathrm{~cm}$. There were then eight plane of motion-object distance combinations. Each combination was given to a subject five times in individually randomized order.

\section{Results and Discussion}

Table 3 presents the perceived distances for the individual subjects as a function of plane of exploration. An ANOVA showed a significant main effect of the experimental manipulation of confining explorations to either the vertical or horizontal plane of motion (vertical mean $=88.8 \mathrm{~cm}$; horizontal mean $=78.3 \mathrm{~cm})[F(1,5)=$ $16.575, p<.01]$. The ANOVA also revealed a signifi- 
Table 3

Perceived Distance (in Centimeters) for Each Subject as a Function of Actual Distance for the Two Planes of Exploration in Experiment 5

\begin{tabular}{clrrrr}
\hline \multirow{2}{*}{ Subject } & & \multicolumn{4}{c}{ Distance } \\
\cline { 3 - 5 } 1 & Plane & 45.7 & 76.2 & 106.7 & 137.2 \\
\hline \multirow{2}{*}{2} & Vertical & 50.8 & 66.8 & 74.9 & 83.1 \\
& Horizontal & 48.8 & 50.8 & 70.2 & 92.0 \\
& Vertical & 83.6 & 93.7 & 97.8 & 121.2 \\
3 & Horizontal & 57.2 & 90.4 & 85.9 & 119.4 \\
& Vertical & 55.1 & 58.7 & 79.0 & 109.7 \\
4 & Horizontal & 39.9 & 52.6 & 74.2 & 101.1 \\
& Vertical & 65.8 & 93.2 & 120.4 & 143.5 \\
5 & Horizontal & 61.2 & 87.4 & 116.3 & 132.6 \\
& Vertical & 93.5 & 78.5 & 116.6 & 137.4 \\
\multirow{2}{*}{6} & Horizontal & 59.2 & 79.0 & 94.5 & 149.4 \\
& Vertical & 34.8 & 54.4 & 80.0 & 132.3 \\
& Horizontal & 31.0 & 41.4 & 70.1 & 75.4 \\
\hline
\end{tabular}

cant main effect of actual object distances on the perceived distances $(57.2,70.6,90.0$, and $116.4 \mathrm{~cm}$, respectively) $[F(3,15)=56.34, p<.0001]$. The interaction of plane of motion and actual object distance was not significant $(F<1)$.

As can be seen in Figure 4, the simple linear regression of the mean vertical data and the mean horizontal data onto the actual object distances accounted for $96 \%$ and $99 \%$ of the variance, respectively. In essence, the restricting of exploration to one of two planes of motion produced a consistent shift in the average perceived distance of the object at each distance from the hand. Importantly, horizontal hand movements during exploration consistently yielded shorter perceived distances than did vertical hand movements for each actual object location on the strand. The data suggest, therefore, that variations in $T$ have greater consequences for distance perception by mechanically generated waves than any potential con-

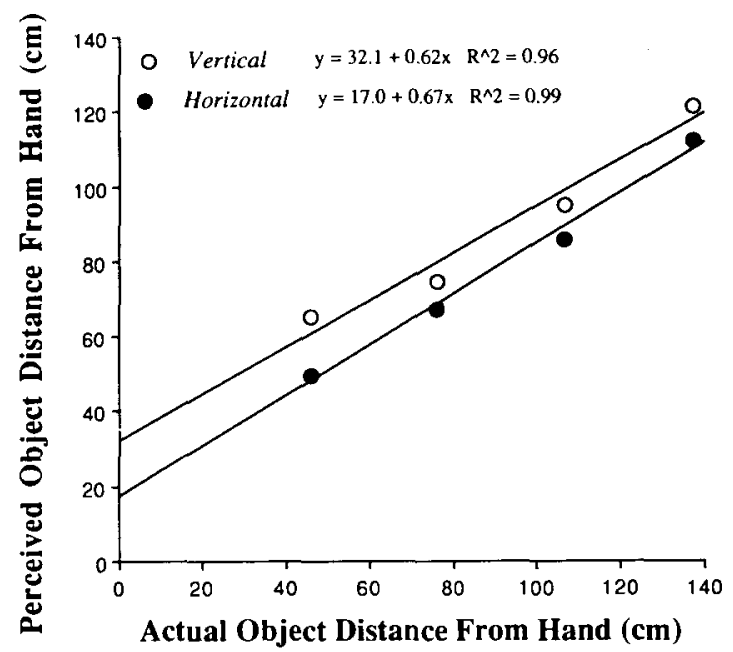

Figure 4. Regression lines for mean perceived distance against actual distance for the two planes of exploration in Experiment 5. tribution by gravity to the perceptually relevant properties of the wave structure transmitted over the strand. The results of Experiment 5, however, do not demonstrate that the influence of gravity is physically negligible on the mechanical waves generated by subjects along the strand. The data suggest only that, relative to the role of tension, gravity does not significantly influence haptic distance perception over a solid elastic medium shaken manually.

\section{EXPERIMENTS 6 AND 7}

The preceding experiments have established that, by manually vibrating a solid medium-specifically, a strand of taut nylon anchored at both ends-a person can perceive the distances of objects attached to the solid medium. Does the subject need to initiate the perturbation of the strand, and does the subject require a reflected wave? Experiments 6 and 7 addressed these questions with respect to the haptic spatial ability under investigation. These experiments were directed at evaluating the effect of experimental conditions that restrict the availability of the transient wave front. Specifically, a subject in these experiments was required to perceive the distance of an object on the basis of only three transient wave fronts. This limit was in sharp contrast to the unlimited availability of transient wave fronts to a subject in the previous experiments, where no restrictions were placed on the period or manner of strand manipulation.

Also, in Experiments 6 and 7, the mechanical event that served to generate the transient wave was the displacement by the experimenter of the object attached to the strand, rather than movement of the strand by the hand of a subject. For these experiments, the wave reaching the hand was an incident wave originating at the point along the strand where the object was attached, not a reflected wave as in Experiments 1-5. The incident wave in Experiments 6 and 7 traversed only the span of the strand from object to hand after the propagating mechanical event. The transient wave as propagated by a subject in Experiments 1-5 traversed the span of the strand medium from the hand to the object and then reflected back from the object to the hand. The same restrictive conditions were in force in both Experiment 6 and Experiment 7; however, Experiment 7 incorporated the additional condition that each subject was given visual feedback after each trial.

Experiments 6 and 7 permitted a partial evaluation of one particular way of conceptualizing how a subject performed the task in Experiments 1-5. Perhaps he or she used the time differential, $\Delta t$, between when the hand initiated the perturbation, $t_{1}$, and when the reflected wave returned to the hand, $t_{2}$. A heuristic strategy available to subjects in Experiments 1-5 would be to base an estimate of object distance from the hand on the relative differences in $\Delta t$ across the various actual object distances. Following this strategy, the larger the $\Delta t$, the further from the hand would be the estimated object location on the strand. It follows, therefore, that if the strand is set in 
motion for a subject, then the basis for temporal comparisons required to employ the heuristic strategy outlined above would be eliminated. In Experiments 6 and 7, the possibility of estimating distance on the basis of $\Delta t$ was excluded because the mechanical waves available to a subject were initiated solely by the experimenter. A systematic dependency of perceived distance on actual distance under these conditions would counter the $\Delta t$ hypothesis.

\section{Method}

Subjects. Eight undergraduate students participated in Experiment 6 , and 7 undergraduate students and 1 graduate student participated in Experiment 7. All students were at the University of Connecticut. By their participation in the experiment, the undergraduates satisfied an introductory psychology course requirement.

Apparatus and Materials. The apparatus and materials of Experiments 6 and 7 were identical to those of Experiments 1 and 2 , with the addition of a $125 \times 250 \mathrm{~cm}$ panel of $1 / 4-\mathrm{in} .(.64-\mathrm{cm})$ plywood covered with centimeter-squared graph paper. This panel provided a reference point for the elevation of the object.

Procedure. In these experiments, a subject grasped firmly the strand but remained immobile at the point of contact while the attached object was set in motion by the experimenter. To set the object in motion, the experimenter would raise it to a set altitude and then release it abruptly such that the elastic recoil would engender a transient wave front. The motion of the object was arrested immediately upon return to its initial position. The object was set in motion in this manner three times in each trial. Pilot work determined that this method of perturbing the strand-object system suppressed the propagation of a standing wave that would otherwise be arrayed along the line if the displaced object's motions were simply allowed to damp out. The height to which the object was raised was marked on a panel next to the strand. This panel was not visible to the subject. The height to which the object was raised prior to release was fixed at $15 \mathrm{~cm}$ across all trials, to ensure that the same work was done against the strand and that the wave fronts generated at each object distance were of the same amplitude. In sum, this procedure served to generate three transient wave fronts originating at the point of attachment for the object in each trial. In Experiment 7, in contrast to Experiment 6, each subject was permitted to check his or her performance visually against the actual object distance at the end of every trial. In all other aspects of procedure, Experiments 6 and 7 followed Experiments 1 and 2 .

Design. The 1.13-kg object was attached at one of seven distances from the point of contact with the haptic web: $45.7,61,76.2,91.4$, $106.7,121.9$, or $137.2 \mathrm{~cm}$. Each of the seven object distances was presented to a subject five times in an individually randomized order.

\section{Results and Discussion}

The mean perceived object distance at each actual object distance was calculated for each subject. For actual distances of $45.7,61,76.2,91.4,106.7,121.9$, and $137.2 \mathrm{~cm}$, the means of the subject means were 69.9 , $80.5,80.3,90.9,94.2,114$, and $126.2 \mathrm{~cm}$, respectively, in Experiment 6, and 67.9, 77.6, 81.3, 95.0, 92.7, 119.4, and $119.5 \mathrm{~cm}$, respectively, in Experiment 7. Regressions of the individual subject and group means on actual object distances are presented in Table $4\left[r^{2}(6)=0.6\right.$, significant at $p<.05$ ]. For only 2 subjects (Subject 7 in Experiment 6, and Subject 8 in Experiment 7) did actual object distance fail to account for a significant amount of the variance in perceived object distance.
Table 4

Simple Regression of Perceived Distance on Actual Distance for the Subjects in Experiments 6 and 7

\begin{tabular}{cccc}
\hline Subject & Intercept & Slope & $r^{2}(6)$ \\
\hline \multicolumn{4}{c}{ Experiment 6} \\
1 & 24.75 & 0.65 & \\
2 & 57.77 & 0.45 & 0.96 \\
3 & 62.79 & 0.48 & 0.91 \\
4 & 40.17 & 0.60 & 0.89 \\
5 & 47.28 & 0.45 & 0.66 \\
6 & 35.85 & 0.69 & 0.92 \\
7 & 61.70 & 0.41 & 0.34 \\
8 & -9.43 & 0.95 & 0.85 \\
& Experiment 7 & \\
1 & 35.81 & 0.72 & 0.92 \\
2 & 29.90 & 0.66 & 0.64 \\
3 & 32.15 & 0.55 & 0.58 \\
4 & 46.97 & 0.59 & 0.77 \\
5 & 51.31 & 0.50 & 0.74 \\
6 & 10.65 & 0.87 & 0.93 \\
7 & 40.68 & 0.52 & 0.67 \\
8 & 71.07 & 0.28 & 0.53 \\
\hline
\end{tabular}

Perceived distance, therefore, exhibited a systematic dependency on actual distance under conditions where selfcontrolled exploration was excluded. Given that the only mechanical waves available to a subject were produced by the experimenter, it would seem that the $\Delta t$ hypothesis advanced above is untenable. The $\Delta t$ hypothesis requires that a subject have the time at which he or she shook the strand available for temporal comparisons that provide the basis for estimates of object distance. The fact that perceived distance remained systematically dependent upon actual distance in spite of the absence of a selfinitiated mechanical event suggests that haptic distance perception over the strand is dependent upon some property or properties of wave structure present in both incident and reflected waves.

A comparison of the regression equations for the group mean data in Experiment $6\left(y=.59 x+40.11, r^{2}=.93\right.$, $p<.001)$ and Experiment $7\left(y=.59 x+39.82, r^{2}=\right.$ $.93, p<.001$ ) with the regression equations for the group mean data in the first two experiments $(y=.73 x+12.01$, $r^{2}=.96, p<.01 ;$ and $y=.83 x+9, r^{2}=.99, p=.001$, respectively) revealed a significant slope difference. Distance perception was less accurate in Experiments 6 and 7 than in the experiments in which manipulation of the strand was unrestricted.

An ANOVA on the data of Experiments 6 and 7 revealed no effect for the between-subject variable of visual feedback $(F<1)$, a significant main effect for the withinsubject variable of object distance $[F(6,14)=43.43$, $p<.0001]$, and no interaction of object distance and visual feedback $(p>.05)$. The ability to locate objects along a vibrating strand under the conditions of Experiment 6 was unmodified by the availability in Experiment 7 of visual information about object distance after each trial. 


\section{GENERAL DISCUSSION}

The series of experiments reported in the present article have demonstrated that a single fibrous strand under high tension, which is contacted manually and vibrated, can support the perception by humans of the distances of (occluded) objects located on the strand. What is the mechanical wave quantity constraining this haptic spatial ability?

An examination of Equation 1 identifies two variables as candidates for indexing the changes in the structure of the energy distribution along the strand specific to perceiving distance: $T$, the measure of the horizontal component of force on the strand, and $v$, the wave's pulse phase velocity, which is independent of both the magnitude of the force at the source that determines the wave amplitude and the shape of the wave (Crawford, 1968; Halliday \& Resnick, 1974). The Appendix details how $\partial^{2} u / \partial x^{2}=\left(1 / v^{2}\right)\left(\partial^{2} u / \partial t^{2}\right)$ is parameterized by object position and identifies the methods for calculating $T$ and $v$. The strand density $\mu$ was held constant in the seven experiments, leaving $T$ and $v$ as the parameters that varied with changes in object location.

An important quantity is the time $\tau$ it takes a transient wave to travel between two points on a segment of the strand under a given tension. It is computed as the distance between the points divided by the appropriate $v$. Thus, as $T$ increases, $v$ increases and $\tau$ between any two points on the segment decreases. $\tau$ is the time scale of the deviation from, and return to, the equilibrium state of the strand. This time scale is informative about the distance of the object from the hand. Given two distances, $d_{1}$ and $d_{2}$, of the same object and two tensions, $T_{1}$ and $T_{2}$, corresponding to the two object-strand configurations, the wave return times over the distances $d_{1}$ and $d_{2}$ will be $d_{1} / v_{1}$ and $d_{2} / v_{2}$, respectively. In that $v$ is proportional to $T$-specifically, to $(T)^{1 / 2}-\tau$ is directly related to $d$ and inversely related to $T$. It is hypothesized, therefore, that

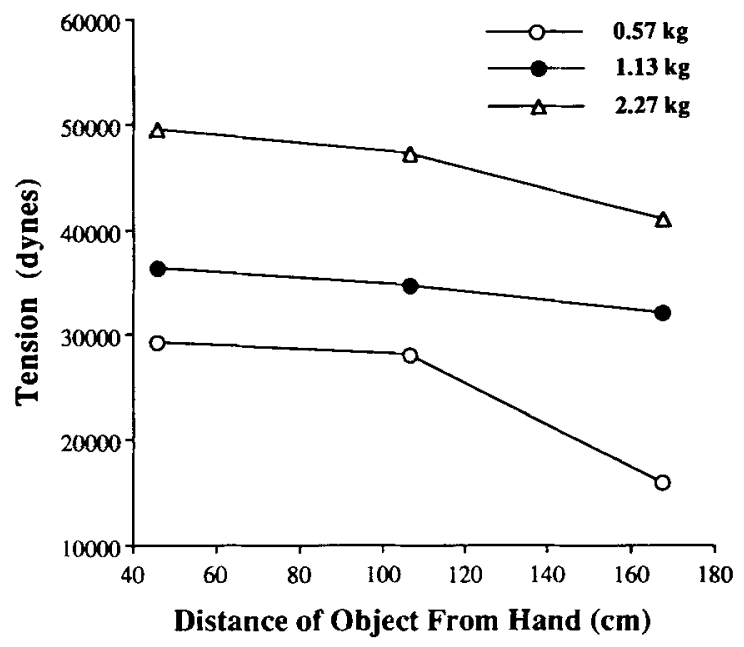

Figure 5. Tension $\boldsymbol{T}$ in strand as a function of object distance from the hand and object mass in Experiment 3.

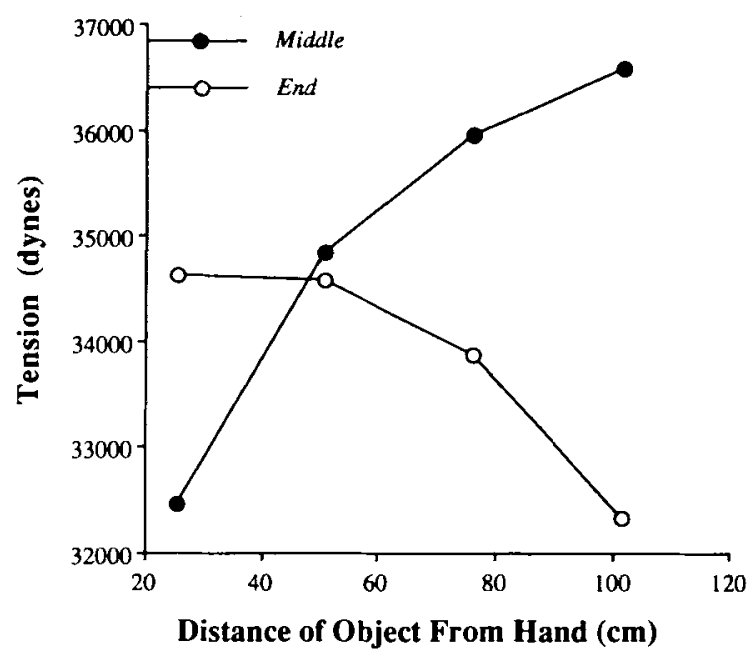

Figure 6. Tension $T$ in strand as a function of object distance from the hand and the position of the hand on the strand (at the end or at the middle) in Experiment 4.

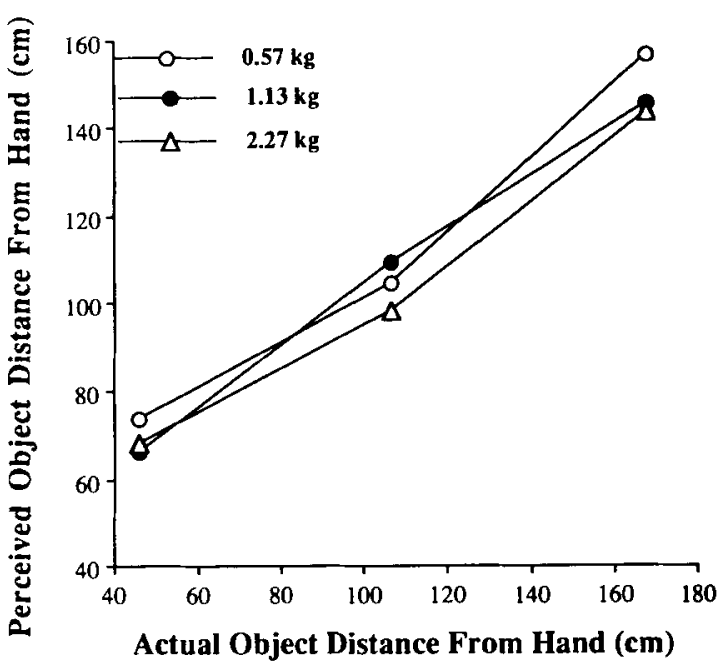

Figure 7. Mean perceived distance against actual distance as a function of object mass in Experiment 3.

perceived distance of an object from the hand is based on the wave travel time $\tau$. According to this hypothesis, perceived distance $\propto d^{\alpha}$ and perceived distance $\propto 1 / T^{\beta}$.

The experiments most suited to evaluating the $\tau$ hypothesis in the form given are Experiments 3 and 4 . In these experiments, the relation between $d$ and $T$ was such that different $T \mathrm{~s}$ were associated with the same $d$ as shown in Figures 5 and 6 . The above analysis of the data of $\mathrm{Ex}$ periment 3 , an experiment in which object mass was manipulated, considered only the quantities of $d$ and mass. The mass variation, it will be recalled, was not significant but approached significance $(p=.07)$, as is evident in Figure 7. Given the methods for calculating $T$ (Equations 3-11 in the Appendix), the data of Experiment 3 can be reanalyzed with the variable $T$ replacing the variable 
of mass. Multiple regression of the 99 individual subject means on $d$ and $T$ revealed a significant effect of $T$ over and above that of $d$. The $r^{2}$ was 0.78 , with $d$ significant at $p<.0001$ and $T$ significant at $p<.05$. Repeating the analysis in logarithmic coordinates yielded beta weights on the two independent quantities of 0.55 and -0.13 , respectively. In brief, the outcomes of the analyses are consistent with the $\tau$ hypothesis as formulated above: Perceived distance went as $d$ to a positive power and perceived distance went as $T$ to a negative power.

The lower right panel of Figure 3 presents the averaged results of Experiment 4, in which the hand was in contact with the strand at an end near the stanchion or at the middle. As is evident from inspection of Figure 3, the mappings of perceived distance to actual distance were markedly different in the two cases. A comparison of Figure 3 showing the perceptual dependency on object distance with Figure 6 showing the $T$ dependency on object distance points to the different relations between $d$ and $T$ as the reason for the different perceptual mappings as a function of hand position. A multiple regression on the averaged data yielded $r^{2}(8)=0.97$, with both $d$ and $T$ significant at $p<.0001$. As a check on the special contribution of $T$, the residual of the simple regression on $d$ was computed; it was found that $T$ accounted for $87 \%$ of the residual. Clearly, in agreement with the $\tau$ hypothesis, both $d$ and $T$ are needed to accommodate the variance in perceived object distance observed in Experiment 4.

In further agreement with the $\tau$ hypothesis, the regression on Experiment 4's data performed in logarithmic coordinates revealed beta weights of 0.50 on $d$ and -3.6 on $T$. These estimates were substantiated by the same multiple regression performed upon the 40 individual subject means ( 20 for hand-at-end and 20 for hand-at-center). Stated as scaling relations, the preceding results yield perceived distance $\propto d^{0.5}$ and perceived distance $\propto T^{-3.6}$. The scaling on $d$ is similar to that found for Experiment 4, whereas the scaling on $T$ is not. It is evident in Figure 6, however, that a single taut strand, as a vibratory medium supporting haptic distance perception, possesses nonlinearities when variations in hand position are juxtaposed with variations in $T$. Parametric experimentation will be needed to establish which power functions hold under which conditions. Understanding such variations in systematic terms will be significant for the general theory of haptic distance perception based on mechanical wave propagation.

\section{CONCLUDING REMARKS}

For many animais, objects and surfaces at a distance from the body can be perceived by means of cutaneous appendages, such as hair, beaks, nails, horns, and vibrissae, and by hand-held probes, and they can be perceived by means of contact with an underlying granular, fibrous, or liquid medium in which waves are propagated. Griffin $(1958 / 1986)$ remarked on the similarities between these distal abilities of touch and the echolocation abilities of bats. He saw echolocation as tool use, in the sense that "a bat fashions useful pulses of sound out of the air it breathes, projects them forward to explore its environment, and listens for echoes that can tell it about what lies ahead" (Griffin, 1958/1986, p. 77). He observed how vibratory movements on a surface and the emission and reception of sound waves in air extended awareness of the environment distal to the animal. The echolocation abilities of humans have been documented for some time (see Griffin 1958/1986, for a historical review), as have their abilities to perceive objects and surfaces at the ends of hand-held probes (e.g., Gibson, 1966; Lotze, 1856/ 1885). Tactile perception's most celebrated student, David Katz (1925/1989), suspected that spatial awareness in humans might be supported by vibrations in the surfaces they contacted. This appreciation of the common exteroceptive abilities of animals and humans based on generating and detecting mechanical waves and mechanical contacts is increased by the present research: It shows that humans, like other animals, can perceive the distances of things by means of vibrations in a solid medium.

\section{REFERENCES}

Barac-Cikoja, D., \& Turvey, M. T. (1991). Perceiving aperture size by striking. Journal of Experimental Psychology: Human Perception \& Performance, 17, 330-346.

BARROWS, W. M. (1915). The reactions of an orb-weaving spider, Epiera sclopetaria $\mathrm{Cl}$, to rhythmic vibrations of its web. Biological Bulletin, 29, 316-332.

BEER, F. P., \& Johnston, E. R. (1972). Vector mechanics for engineer: Statics. New York: McGraw-Hill.

Bristowe, W. S. (1958). The world of the spider. London: Collins. Brownell, P. H. (1984). Prey detection by the sand scorpion. Scientific American, 251(6), 86-97.

BURGESS, J. W. (1975). The sheet web as a transducer, modifying vibration signals in social spider colonies of Mallos gregalis. Neuroscience Abstracts, 1, 557.

Burgess, J. W. (1976). Social spiders. Scientific American, 234(3), 100-106.

BURGEss, J. W. (1979). Web-signal processing for tolerance and group predation in the social spider Mallos gregalis Simon. Animal Behaviour, 27, 157-164.

Burgess, J. W., W Witt P. N. (1976). Spider webs: Design and engineering. Interdisciplinary Science Reviews, 1, 322-335.

Burton, G., Turvey, M. T., Solomon, H. Y. (1990). Can shape be perceived by dynamic touch? Perception \& Psychophysics, 48 , 477-487.

Carello, C., Fitzpatrick, P., Domaniewicz, I., Chan, T.-C., \& TURVEY, M. T. (1992). Effortful touch with minimal movement. Journal of Experimental Psychology: Human Perception \& Performance, 18, 290-302.

Carello, C., Fitzpatrick, P., Turvey, M. T. (1992). Haptic probing: Perceiving the length of a probe and the distance of a surface probed. Perception \& Psychophysics, 51, 580-598.

Chan, T.-C., \& Turvey, M. T. (1991). Perceiving the vertical distance of surfaces by means of a hand-held probe. Joumal of Experimental Psychology: Human Perception \& Performance, 17, 347-358.

Crawford, F. S. (1968). Waves. New York: McGraw-Hill.

Den Hartog, J. P. (1934). Mechanical vibrations. New York: McGraw-Hill.

GiBson, J. J. (1966). The senses considered as perceptual systems. Boston: Houghton Mifflin.

GRIFFIN, D. R. (1986). Listening in the dark. Ithaca, NY: Comstock Publishing Associates. (Original work published 1958) 
Halliday, D., \& Resnick, R. (1974). Fundamentals of physics. New York: Wiley.

KATZ, D. (1989). The world of touch (L. E. Kreuger, Trans.). Hillsdale, NJ: Erlbaum. (Original work published 1925)

LoTzE, R. H. (1885), Mikrokosmos, Vol. IT (4th ed., E. Hamilton \& E. E. Constance Jones, Trans.). Edinburgh: T \& T Clark. (Original work published 1856)

MoRSE, P. M. (1948). Vibration and sound. New York: McGraw-Hill.

Pagano, C. C., \& Turvey, M. T. (1992). Eigenvectors of the inertia tensor and perceiving the orientation of a hand-held object by dynamic touch. Perception \& Psychophysics, 52, 617-624.

PARry, D. A. (1965). The signal generated by an insect in a spider's web. Journal of Experimental Biology, 43, 185-192.

PedhazUR, E. J. (1982). Multiple regression in behavioral research. New York: Holt, Rinehart \& Winston.

Seto, W. W. (1964). Mechanical vibrations. New York: McGraw-Hill.

Solomon, H. Y., \& TURVEY, M. T. (1988). Haptically perceiving the distances reachable with hand-held objects. Journal of Experimental Psychology: Human Perception \& Performance, 14, 404-427.

Solomon, H. Y., Turvey, M. T., \& Burton, G. (1989a). Gravitational and muscular variables in perceiving rod extent by wielding. Ecological Psychology, 3, 265-300.

Solomon, H. Y., Turvey, M. T., \& Burton, G. (1989b). Perceiving rod extent by wielding: Haptic diagonalization and decomposition of the inertia tensor. Journal of Experimental Psychology: Human Perception \& Performance, 15, 58-68.

SYmoN, K. (1971). Mechanics. New York: Addison-Wesley.

SZLEP, R. (1964). Change in the response of spiders to repeated web vibrations. Behaviour, 23, 203-239.

Turvey, M. T., Burton, G., Pagano, C., Solomon, H. Y., Runeson, S. (1992). Role of the inertia tensor in perceiving object orientation by dynamic touch. Joumal of Experimental Psychology: Human Perception \& Performance, 18, 714-727.

Von Frisch, K. (1974). Animal architecture. New York: Harcourt Brace Jovanovich.

WalcotT, C. (1963). The effect of the web on vibration sensitivity in the spider Acharanea tepidariorum Koch. Journal of Experimental Zoology, 141, 191-244.

WALCoTt, C. (1969). A spider's vibration receptor: Its anatomy and physiology. American Zoologist, 9, 133-144.

WiTt, P. (1975). The web as a means of communication. Bioscience Communication, 1, 7-23.

\section{APPENDIX \\ Basic Vibratory Mechanics of the Single-Strand Haptic Web as a "Stretched String"}

Under certain conditions, a single strand may be treated as a one-dimensional body of uniform density and finite size, without the characteristic hyperbolic curvature of the catenary. This treatment as a "stretched string" is possible whenever the strand is held taut at anchor points, at a fixed distance apart, and under tension sufficient to eliminate discernible sag, yet not in excess of the elastic limits of the strand (Crawford, 1968; Morse, 1948). The impulsive application of force to a stretched string (as occurs when a subject shakes the strand) propagates a transient transverse wave, one that displaces the strand perpendicularly to the direction of propagation along the strand's length. Waves traveling from the source are termed incident. All incident traveling waves in the strand are reflected from the boundaries (anchor points) and from any high-density inhomogeneity, such as a metal object attached to the strand. Each such reflection gives rise to a reflected wave traveling in the strand in the opposite direction back toward the point of application of force.

The velocity with which both incident and reflected waves traverse the strand is termed the pulse phase velocity, $v$. The pulse phase velocity of any transient (impulsive) wave front propagated in the strand depends solely on the rest-state force structure of the strand, but is independent of both magnitude of the force at the source (that determines wave amplitude) and the shape of the wave-sinusoidal, sawtooth, square, and so on. Specifically, for all incident and reflected waves propagated in a stretched string, $v$ goes as the tension $(T)$ on the line (the horizontal force component) divided by the linear density $\mu$ (the mass per unit length of the strand) to the $1 / 2$ power,

$$
\nu=(T / \mu)^{1 / 2} \text {. }
$$

Both $T$ and $\mu$ are directly measurable for any configuration of taut strand with attached object, as long as the mathematical relation between the deformation and the restoring force is one of simple proportionality, as is the case when the force(s) imposed do not exceed the elastic limits of the strand. Such a relation is expressed by a linear equation that holds for all kinetic exchanges on the strand that are not of sufficient magnitude to stretch the strand into the region of hysteresis ("elastic fatigue"). Passing through this region leads to an abrupt redistribution of potentials along the strand-a break in the line. Obviously, any strand subjected to forces of such catastrophic magnitudes cannot be modeled as a stretched string (Den Hartog, 1934; Halliday \& Resnick, 1974; Morse, 1948).

The single-strand haptic web with an attached object has a reststate force structure that can be exhaustively characterized by a free-body (force) diagram. Following this method, an inertial reference frame is selected that places the origin at the junction of the strand segments, running from both anchor points to the point on the strand where the object is attached (Figure 8a). The point of attachment is taken to be the "body" in this reference frame. The body is in static equilibrium, remaining at rest under the action of the three forces imposed on it by the two strand segments (A and $\mathrm{B}$ ) and the weight ( $W$ ) of the object. As the body is unaccelerated, all forces acting upon it lie in a single plane and are captured by the vectors $F_{A}, F_{B}$, and $F_{C}$, where

$$
F_{A}+F_{B}+F_{C}=0 .
$$

This is tantamount to saying that, from the standpoint of the requirements of mechanical analysis, the strand has only two dimensions of relevance. Even the cross-sectional area of the strand is reduced in dimensionality when it is subsumed under the index of the strand material's mass per unit length, the "linear density" term that is the denominator in the calculated values of pulse phase velocity (Halliday \& Resnick, 1974). Choosing the $x$ - and $y$-axis as shown (Figure 8b), this vector equation can be rewritten as three scalar equations:

$$
\begin{gathered}
F_{A x}+F_{B x}=0, \\
F_{A y}+F_{B y}+F_{C y}=0,
\end{gathered}
$$

and

$$
F_{A z}=F_{B z}=F_{C z}=0
$$

(as all the vectors lie in the $x y$ plane they have no $z$-components). This follows from the decomposition of the vectorial expression of Newton's second law, $\boldsymbol{F}=m \mathrm{~m}$, into the three scalar equations, $F_{x}=m a_{x}, F_{y}=m a_{y}$, and $F_{z}=m a_{2}$, which relate the components of the resultant force to the corresponding components of acceleration for the mass $m$. This relationship holds only for systems for which the resultant force $\boldsymbol{F}$ is zero. Any system with the translational particle dynamics expressed by these relations is termed static. 
a

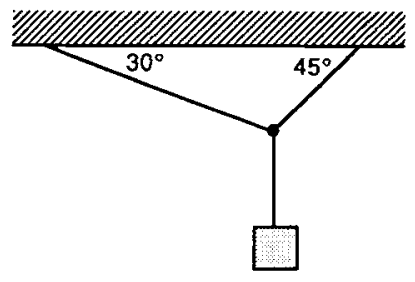

b

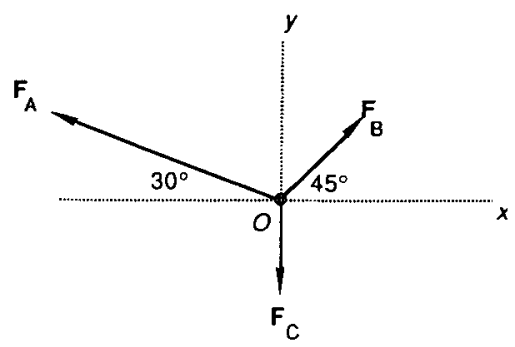

Figure 8. Calculating the forces on a stretched string (see text for details). In this example, Angle $A=30^{\circ}$, Angle $B=45^{\circ}$.

With the above framework in place, and knowing the mass of the object attached at any given point along the strand, the tension along both the strand segments running to either anchor point is calculable from the direct measurement of the angles of deflection, given

$$
\begin{aligned}
& F_{A x}=-F_{A} \cos \text { Angle A, } \\
& F_{A y}=F_{A} \text { sin Angle A, }
\end{aligned}
$$

and

$$
\begin{aligned}
& F_{B x}=F_{B} \cos \text { Angle B, } \\
& F_{B y}=F_{B} \sin \text { Angle B, }
\end{aligned}
$$

while the last component is simply

$$
F_{C y}=-F_{C}=-W \text {. }
$$

Substituting these known quantities directly into the simultaneous scalar equations of the static strand-object system, the tensions are easily derived using the elementary algebraic technique of Gaussian Back Substitution (Beer \& Johnston, 1972; Halliday $\&$ Resnick, 1974). The measured angles of deflection for each of the strand segments (A and B) at the point of attachment for the object, in combination with the measure of tension, capture the local gradient elastic potentials against which work must be done to set the strand in motion. As stated earlier, this exhaustively describes the rest-state force structure of the "haptic web" under the load of an attached object.

It is important to note that this static equilibrium state is the high-symmetry state (relative to the distribution of potentials along the strand) that is broken when a subject's exploratory manipulations propagate a transient wave in the single-strand haptic web. (Pilot work demonstrated that subjects were completely unable to perceive object distance when only passive contact was made with the static strand-object system. This fact supports the characterization of the relative perceptual transparency of the different symmetry states of the single-strand haptic web and directs efforts toward understanding active exploratory contact with the strand.)

Additionally, it is worth noting that the propagation of a transient wave in the single-strand haptic web represents the only low-symmetry structured array available to underwrite vibratory perception, as the application of force to the strand on a continuous basis results in the propagation of a symmetrical standing wave (Crawford, 1968; Morse, 1948). The standing wave is the dynamic equilibrium state of the strand under continuous forcing. As such, it represents a maximally symmetrical redistribution of potentials along the strand, which marks the transition from the static high-symmetry state of the strandobject system to another high-symmetry state rather than to a break in symmetry. This has the consequence that the force structure along the strand (and, therefore, what is available for detection by an organism) is locally stable and uninformative. $\mathrm{Pi}$ lot work demonstrated that, when a subject made contact with the strand in a manner such that a standing wave was propagated, perception of object distance was poor. It is also the case that when a subject was allowed to freely select his or her manner of exploration he or she inevitably settled on the impulsive application of force at irregular intervals-the exploratory style that propagates a transient wave front. The literature on the predatory behaviors of orb-weaver spiders reveals that these spiders similarly agitate the transmission strand of their webs in an impulsive manner in order to detect the presence and location of potential prey (Von Frisch, 1974).

(Manuscript received November 22, 1991; revision accepted for publication May 19, 1992.) 